\title{
Outcomes of SARS-CoV-2 infection in patients with celiac disease: a multicenter research network study
}

\author{
Yousaf Bashir Hadia, Amir Humza Sohail'b, Dhairya A. Lakhanic, Syeda Fatima Naqvia, Justin T. Kupeca, \\ Asad Pervez ${ }^{\mathrm{a}}$
}

West Virginia University, Morgantown; NYU Langone, Long Island, Mineola, NY, USA

\section{Abstract}

\begin{abstract}
Background Celiac disease (CD) is associated with an increased risk for respiratory infections and severe outcomes. No data have been reported in the scientific literature regarding the outcomes of COVID-19 in this population. The aim of this study was to report matched clinical outcomes in a large cohort of 930 patients with COVID-19 in the setting of known CD.
\end{abstract}

Methods Analysis of a multicenter research network TriNETX was performed, including COVID-19 patients aged more than 16 years. Outcomes of COVID-19-positive patients with concurrent $\mathrm{CD}$ were compared with a propensity-matched cohort of patients without $\mathrm{CD}$.

Results A total of 341,499 patients with SARS-CoV-2 infection were identified on the research network: $930(0.27 \%)$ with $\mathrm{CD}$ and 340,569 (99.73\%) without CD. In the 30- and 60-day periods post SARS-CoV-2 infection, $12(1.29 \%)$ and $13(1.40 \%)$ deaths, respectively, were reported in the $\mathrm{CD}$ group. Fewer patients in the $\mathrm{CD}$ group reached the composite outcome of either mechanical ventilation or mortality at 60 days (risk ratio $0.58,95 \%$ confidence interval 0.36-0.95). After propensity matching, no difference in clinical outcomes was observed.

Conclusion Our data suggest that patients with CD are not at increased risk of COVID-19-related morbidity or mortality.

Keywords SARS-CoV-2, COVID-19, celiac disease, morbidity, mortality

Ann Gastroenterol 2022; 35 (X): 1-5

\section{Introduction}

Celiac disease $(\mathrm{CD})$ is a chronic immune-mediated glutendependent enteropathy, with a global prevalence of $1.4 \%$ based on serologic testing and $0.7 \%$ based on biopsy results [1]. It is associated with an increased risk for sepsis, respiratory infections and severe outcomes with conditions such as pneumococcal disease [2], varicella zoster [3], and influenza [4,5]. There is some evidence to suggest that this increased risk persists even among asymptomatic patients after adoption of a gluten-free diet $[4,6]$. Interestingly, this increased susceptibility results from the pathophysiology of $\mathrm{CD}$, rather than from immunosuppressive therapy, as seen

${ }^{\mathrm{a}}$ Department of Medicine, West Virginia University, Morgantown (Yousaf Bashir Hadi, Syeda Fatima Naqvi, Justin T. Kupec, Asad Pervez); ${ }^{b}$ Department of Surgery, NYU Langone, Long Island, Mineola, NY (Amir Humza Sohail); 'Department of Radiology, West Virginia University, Morgantown (Dhairya A. Lakhani), USA

Conflict of Interest: None

Correspondence to: Amir H. Sohail, MD, MSc, Department of Surgery, NYU Langone, Long Island, Mineola, 11501, USA, e-mail: amir.sohail@ nyulangone.org

Received 9 July 2021; accepted 15 November 2021; published online 2 February 2022

DOI: https://doi.org/10.20524/aog.2022.0691

๑) 2022 Hellenic Society of Gastroenterology in other immune-mediated conditions, such as inflammatory bowel disease [7]. In theory, this intrinsically high susceptibility, compounded with malnutrition and immunosuppressive medical management, may put $\mathrm{CD}$ patients at risk of life-threatening disease in the SARS-CoV-2 pandemic [8].

As the SARS-CoV-2 pandemic evolves, it is paramount that outcomes in commonly susceptible patient subpopulations be studied to allow risk stratification and guide therapy. However, there is a paucity of scientific evidence regarding the outcomes of COVID-19 in patients with $\mathrm{CD}$. To address this gap, we analyzed the clinical outcomes among a large cohort of 930 patients with COVID-19 in the setting of known CD. Outcomes were compared with a propensitymatched cohort of COVID-19 patients without CD.

\section{Patients and methods}

\section{Data source}

A retrospective cohort study was conducted utilizing the multiinstitutional research network TriNETX (Cambridge, MA, USA) platform. TriNETX is a federated research network that includes more than 40 healthcare organizations in the USA, providing real-time access to the healthcare records. It includes $>40$ million patients from the healthcare organizations, in a de-identified 
fashion. The data are directly retrieved from the electronic health record management systems (EHRs) of participating organizations. These organizations are large academic centers that operate both tertiary care and satellite outpatient office locations.

Clinical variables (facts) are derived directly through a builtin natural language processing system that extracts variables from clinical documents. Robust quality assurance is achieved at the time of extraction from EHRs before inclusion in this database. This interface only provides aggregate counts and statistical summaries to protect personal health information and ensures data remain de-identified at all levels of retrieval and dissemination.

TriNetX has received a waiver from Western IRB as it only provides de-identified information. At our institution West Virginia University Clinical and Translational Science Institute (WVU CTSI) manages the TriNetX platform and provides access to the end-users.

\section{Patient selection}

Centers for Disease Control and Prevention COVID-19 coding guidance was used to identify COVID-19 cases. The International Classification of Diseases, Ninth Revision and Tenth Revision, Clinical Modification (ICD-10-CM) codes and Logical Observation Identifiers Names and Codes codes for positive laboratory tests were used. This search strategy has been used previously in published data from the TriNetX. The codes are illustrated in Supplementary Table 1. A real time search and analysis was conducted on the TriNetX platform and updated through February 1, 2021, to identify patients aged more than 16 years with SARS-CoV-2 infection. Patients were then classified into a CD cohort and a non-CD cohort, based on a previous diagnosis of CD. This study method has been validated previously $[9,10]$.

\section{Study duration}

Enrolment between January 20, 2020, and February 01, 2021 was required for inclusion in the study cohort. January 20, 2020, was chosen as it was the date of diagnosis of the first case of SARS-CoV-2 infection in the USA. February 01, 2021, was chosen to ensure 60 days of follow up for all included patients, since the primary study endpoint was a composite outcome at 60 days from diagnosis.

\section{Study outcomes}

The primary study outcome was a composite event of death or requirement for mechanical ventilation during 30- and 60day periods from the index event. Other outcomes included death, hospitalization, and need for critical care during the 30- and 60-day follow-up periods.

\section{Study definitions for data aggregation}

Index event: Diagnosis of COVID 19 by criteria defined above.
Time windows: Study outcomes were assessed at 30 and 60 days from the index event and included the day of the index event. Baseline characteristics for patients were considered until the day of COVID-19 diagnosis or first positive COVID-19 test result.

\section{Statistical analysis}

The TriNetX platform was used to conduct all analyses. The chi-square test and $t$-test were used for univariate analyses. Propensity score matching was performed 1:1 with age, race, diabetes, hypertension, chronic lung diseases, nicotine dependence, heart failure, ischemic heart disease, body mass index, and sex as covariates, and a propensity score-matched control group of patients without CD was identified. The 1:1 matching was performed based on the propensity scores generated by using greedy nearest neighbor algorithms with a caliper width of 0.1 pooled standard deviations; further details are illustrated in Supplementary Table 1. The balance on covariates was assessed using the standardized mean difference, and absolute values $>0.1$ were considered positive for residual imbalance. A 2-sided alpha of less than 0.05 was defined $a$ priori for statistical significance. Risk ratios (RR) with $95 \%$ confidence intervals (CI) were calculated for all analyses. The TriNetX platform utilizes input matrices of user identified covariates and conducts logistic regression analysis to obtain propensity scores for individual subjects. TriNetX randomizes the order of rows in order to eliminate bias resulting from nearest neighbor algorithms.

\section{Results}

\section{Study population}

We identified a total of 341,499 patients with SARS-CoV-2 infection on the research network: $930(0.27 \%)$ with CD and 340,569 (99.73\%) without CD. Baseline characteristics and associated comorbid conditions are presented in Table 1. Comorbidities, including hypertension, diabetes mellitus and lower respiratory diseases, were more common in patients with $\mathrm{CD}$, as was Caucasian race and female sex (all $\mathrm{P}<0.05)$.

\section{Clinical outcomes}

Eighty-one patients with CD required hospitalization (8.71\%). In the 30- and 60-day periods post-SARS-CoV-2 infection, $12(1.29 \%)$ and $13(1.40 \%)$ deaths, respectively, were reported in the CD group. The composite outcome was reached by 16 (1.72\%) in the CD group, at both 30-and 60-day time points (Table 2). In the crude unmatched analysis, no difference was observed between the $\mathrm{CD}$ and non-CD cohorts in rates of hospitalization, need for critical care, acute kidney injury or mortality at 30-and 60-day follow up; however, fewer patients in the $\mathrm{CD}$ group reached the composite outcome at 60 days (RR 0.58, 95\%CI 0.36-0.95).

After propensity matching, there was no difference between the 2 cohorts in the rates of composite endpoint at 30 days (RR 0.73 , 95\%CI 0.38-1.38) and 60 days (RR 0.70, 95\%CI 0.37-1.31), 30-day (RR 0.80, 95\%CI 0.38-1.70), and 60-day (RR 0.87, 95\%CI 0.42 - 
SARS-CoV-2 and celiac disease 3

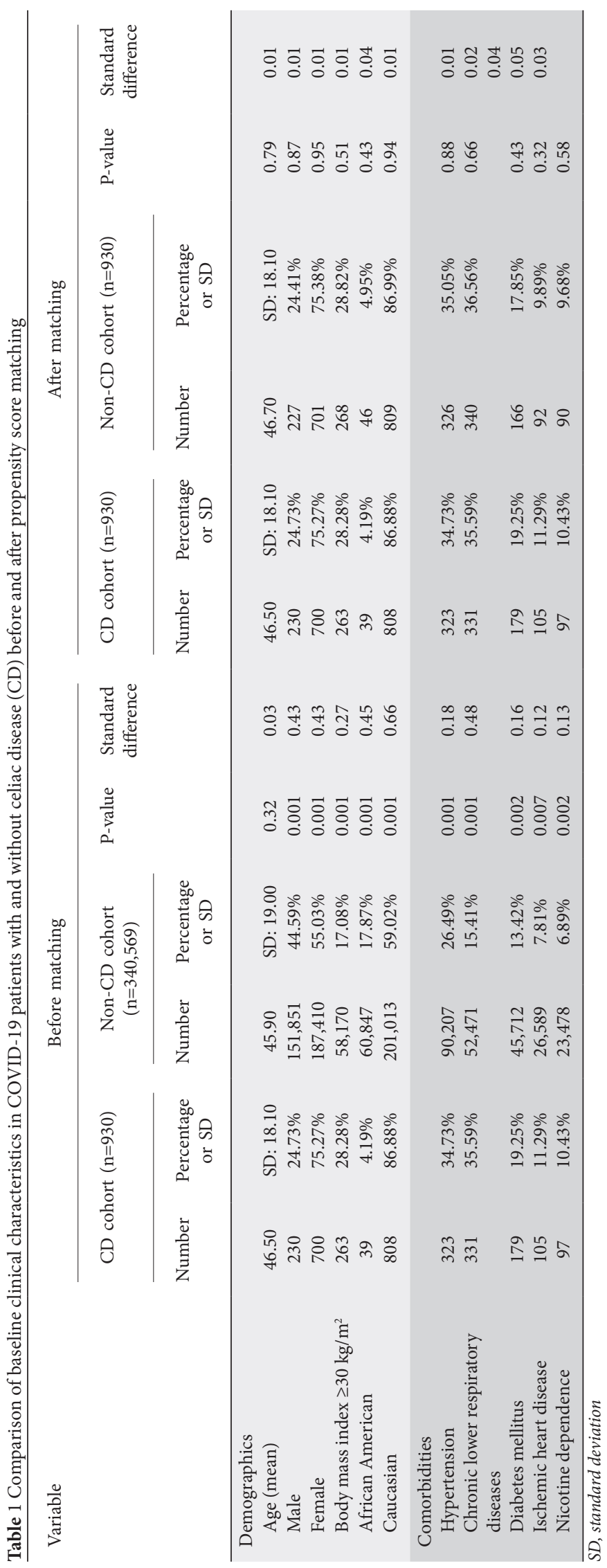


1.81) mortality, need for hospitalization (RR 1.00, 95\%CI $0.75-$ 1.34), critical care requirements (RR $0.89,95 \% \mathrm{CI} 0.52-1.53$ ), or acute renal failure (RR 1.39, 95\%CI 0.84-2.27), as shown in Table 2.

\section{Discussion}

Our study shows that patients with $\mathrm{CD}$ do not have a significantly increased risk for worse outcomes, including mortality, need for hospitalization, critical care services and acute kidney injury. In fact, in the crude analysis, patients with $\mathrm{CD}$ were at lower risk of reaching the composite poor outcome at 60-day follow up (RR 0.58, 95\%CI 0.36-0.95). This association did not hold true after propensity matching, which may indicate that the lower risk among CD patients was driven by a higher proportion of females (75.27\%) and Caucasians (86.88\%) in the CD group, known to have better outcomes with COVID-19 [8,11]. It is noteworthy that only $10 \%$ of patients required hospitalization in the CD cohort. This is striking, given the well-documented evidence for worse outcomes with respiratory infections in CD patients $[3,4,12]$.

As the COVID-19 pandemic continues to evolve, there is a dire need to identify risk factors for poor outcomes with COVID-19 to optimize risk stratification for prevention, vaccine administration, surveillance and therapeutic measures, especially in high-risk populations. Evidence on outcomes of COVID-19 infection shows that patients with a weak immune system, such as the elderly population or those with multiple comorbid conditions, tend to have a higher case fatality rate [13]. Further, data show that transplant populations, especially patients on long-term immunosuppressive therapy, tend to have particularly worse outcomes with COVID-19 $[9,14]$. Thus, it could be argued theoretically that $\mathrm{CD}$, with its baseline alterations in afflicted individuals' immune profile, immunosuppressive therapy, and other well-documented data on higher predisposition and poor outcomes with respiratory infections (e.g., pneumococcal pneumonia, tuberculosis, and influenza), should alter the disease course of COVID-19 in the CD population for the worse [2-5]. Interestingly, another postulation is that since the immunemediated cytokine response is primarily responsible for the damage resulting from COVID-19, CD patients, with their weaker immune systems, may fare better with COVID-19 infection [15].

Lionetti et al [15] and Zhen et al [16] found no increased risk for contracting COVID-19 in patients with CD. Likewise, Zingone et al conducted a cross-sectional study at the height of the pandemic in Italy and found that, among a cohort of 138 CD patients, none had been diagnosed with COVID-19 [17]. Data from Sweden also corroborate our findings: Lebwohl et al showed that CD patients with COVID-19 were not at a significantly higher risk of hospitalization, severe COVID-19 or all-cause mortality [18]. Importantly, results from the Surveillance Epidemiology Under Research Exclusion for Celiac Disease (SECURE-CELIAC) [19], an international, de-identified database comprising 123 COVID-19 cases among patients with CD from 21 countries, also noted comparable hospitalization (12\%) and case fatality rates (2.5\%) to the general population: $20 \%$ hospitalization rate $[20,21]$ and $1-11 \%$ case fatality rate $[22,23]$.

Our study is one of the first to investigate the outcome of COVID-19 in patients with CD. Propensity matching and a

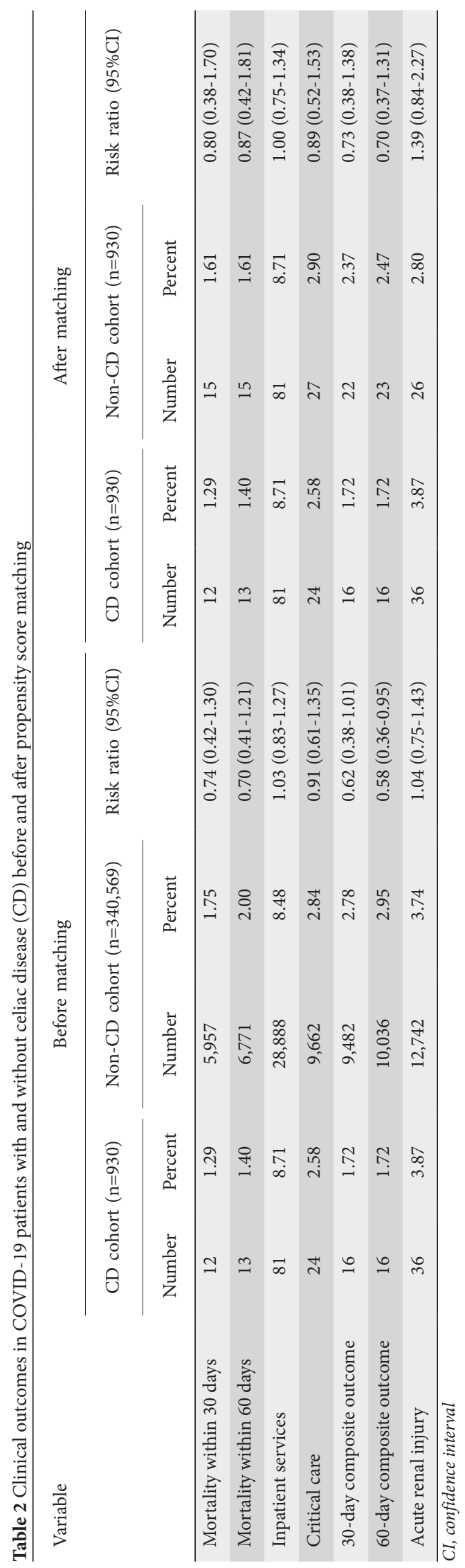


large cohort support the reliability of our results. Importantly, the multicenter nature of our study also increased the heterogeneity in the included CD patient population, extending the generalizability of our conclusions. The major limitations of our study include biases inherent to studies based on electronic healthcare records, including misclassification bias. Residual confounding from imprecisely measured and unmatched variables, for instance physical activity, cannot be excluded.

In conclusion, our results show that patients with $\mathrm{CD}$ are not at higher risk of short-term COVID-19 related morbidity or mortality. The clinical course of COVID-19 in CD patients is not sufficiently worse to warrant a higher level of inpatient or critical care services compared to COVID-19 patients without CD. However, further longitudinal studies investigating longterm disease outcomes, particularly respiratory complications, in CD patients are required to bridge gaps in data and ensure optimal long-term surveillance and intervention.

\section{Summary Box}

\section{What is already known:}

- Immunocompromised populations, such as transplant patients, often have worse outcomes with COVID-19

- Celiac disease is associated with an increased risk of infection and poor outcomes with respiratory infections

- There are only limited data on outcomes of COVID-19 in patients with celiac disease

\section{What the new findings are:}

- The hospitalization rate for COVID-19 infection in patients with celiac disease was $8.71 \%$

- The 30- and 60-day post-COVID-19 infection mortality rates in a celiac disease population were $1.29 \%$ and $1.40 \%$, respectively

- There was no difference in 30- or 60-day mortality, need for hospitalization, critical care requirements, or acute renal failure in patients with celiac disease compared to the general population

\section{References}

1. Singh P, Arora A, Strand TA, et al. Global prevalence of celiac disease: systematic review and meta-analysis. Clin Gastroenterol Hepatol 2018;16:823-836.

2. Thomas HJ, Wotton CJ, Yeates D, Ahmad T, Jewell DP, Goldacre MJ. Pneumococcal infection in patients with coeliac disease. Eur $J$ Gastroenterol Hepatol 2008;20:624-628.

3. Ludvigsson JF, Choung RS, Marietta EV, Murray JA, Emilsson L. Increased risk of herpes zoster in patients with coeliac disease - nationwide cohort study. Scand J Public Health 2018;46:859-866.

4. Mårild K, Fredlund H, Ludvigsson JF. Increased risk of hospital admission for influenza in patients with celiac disease: a nationwide cohort study in Sweden. Am J Gastroenterol 2010;105:2465-2473.

5. Walters JRF, Bamford KB, Ghosh S. Coeliac disease and the risk of infections. Gut 2008;57:1034-1035.

6. Emilsson L, Lebwohl B, Green PH, Murray JA, Mårild K, Ludvigsson JF. Mucosal healing and the risk of serious infections in patients with celiac disease. United European Gastroenterol J 2018;6:55-62.

7. Brenner EJ, Ungaro RC, Gearry RB, et al. Corticosteroids, but not TNF antagonists, are associated with adverse COVID-19 outcomes in patients with inflammatory bowel diseases: results from an international registry. Gastroenterology 2020;159:481-491.

8. Golestaneh L, Neugarten J, Fisher M, et al. The association of race and COVID-19 mortality. EClinicalMedicine 2020;25:100455.

9. Hadi YB, Naqvi SFZ, Kupec JT, Sofka S, Sarwari A. Outcomes of COVID-19 in solid organ transplant recipients: a propensitymatched analysis of a large research network. Transplantation 2021;105:1365-1371.

10. Hadi YB, Lakhani DA, Naqvi SF, Fatima NU, Sarwari AR. Outcomes of SARS-CoV-2 infection in patients with cystic fibrosis: a multicenter retrospective research network study. Respir Med 2021;188:106606.

11. Gebhard C, Regitz-Zagrosek V, Neuhauser HK, Morgan R, Klein SL. Impact of sex and gender on COVID-19 outcomes in Europe. Biol Sex Differ 2020;11:29.

12. Comba A, Atan D. Evaluation of nasal mucociliary clearance time in children with celiac disease. Int J Pediatr Otorhinolaryngol 2020;133:109936.

13. Onder G, Rezza G, Brusaferro S. Case-fatality rate and characteristics of patients dying in relation to COVID-19 in Italy. JAMA 2020;323:1775-1776.

14. Bhoori S, Rossi RE, Citterio D, Mazzaferro V. COVID-19 in longterm liver transplant patients: preliminary experience from an Italian transplant centre in Lombardy. Lancet Gastroenterol Hepatol 2020;5:532-533.

15. Lionetti E, Fabbrizi A, Catassi C. Prevalence of COVID-19 in Italian children with celiac disease: a cross-sectional study. Clin Gastroenterol Hepatol 2021;19:1075.

16. Zhen J, Stefanolo JP, de la Paz Temprano M, et al. The risk of contracting COVID-19 is not increased in patients with celiac disease. Clin Gastroenterol Hepatol 2021;19:391-393.

17. Zingone F, D'Odorico A, Lorenzon G, Marsilio I, Farinati F, Savarino EV. Risk of COVID-19 in celiac disease patients. Autoimmun Rev 2020;19:102639.

18. Lebwohl B, Larsson E, Söderling J, et al. Risk of severe COVID-19 in patients with celiac disease: a population-based cohort study. Clin Epidemiol 2021;13:121-130.

19. Uche-Anya E, Husby S, Kaplan GG, Underwood FE, Green PHR, Lebwohl B. An international reporting registry of patients with celiac disease and COVID-19: initial results from SECURECELIAC. Clin Gastroenterol Hepatol 2021;19:2435-2437.

20. CDC COVID-19 Response Team. Preliminary estimates of the prevalence of selected underlying health conditions among patients with coronavirus disease 2019 - United States, February 12-March 28, 2020. MMWR Morb Mortal Wkly Rep 2020;69:382-386.

21. Petersen E, Koopmans M, Go U, et al. Comparing SARS-CoV-2 with SARS-CoV and influenza pandemics. Lancet Infect Dis 2020;20:e238-e244.

22. Wu Z, McGoogan JM. Characteristics of and important lessons from the coronavirus disease 2019 (COVID-19) outbreak in China: summary of a report of 72314 cases from the Chinese Center for Disease Control and Prevention. JAMA 2020;323:1239-1242.

23. Sudharsanan N, Didzun O, Bärnighausen T, Geldsetzer P. The contribution of the age distribution of cases to COVID-19 case fatality across countries: a nine-country demographic study. Ann Intern Med 2020;173:714-720. 


\section{Supplementary material}

Supplementary Table 1 ICD 10 and LOINC codes used to identify study participants

LOINC Codes

\begin{tabular}{lcl}
\hline Number & LOINC codes & Description \\
\hline 1 & $94533-7$ & SARS coronavirus 2 N gene [Presence] in Respiratory specimen by NAA with probe detection \\
\hline 2 & $94534-5$ & SARS coronavirus 2 RdRp gene [Presence] in Respiratory specimen by NAA with probe detection \\
\hline 3 & $41458-1$ & SARS coronavirus RNA [Presence] in Unspecified specimen by NAA with probe detection \\
\hline 4 & $94309-2$ & SARS coronavirus 2 RNA [Presence] in Unspecified specimen by NAA with probe detection \\
\hline 5 & $94531-1$ & SARS Coronavirus 2 RNA panel - Respiratory specimen by NAA with probe detection \\
\hline 6 & $94506-3$ & SARS coronavirus 2 IgM Ab [Units/volume] in Serum or Plasma by Immunoassay \\
\hline 7 & $94500-6$ & SARS coronavirus 2 RNA [Presence] in Respiratory specimen by NAA with probe detection \\
\hline 9 & $94315-9$ & SARS coronavirus 2 E gene [Presence] in Unspecified specimen by NAA with probe detection \\
\hline 10 & $94316-7$ & SARS-CoV-2 (COVID19) N gene [Presence] in Unspecified specimen by NAA with probe detection \\
\hline
\end{tabular}

ICD 10 codes

\begin{tabular}{lll}
\hline 1 & K 90.0 & Celiac disease \\
\hline 2 & B34.2 & Coronavirus infection, unspecified \\
3 & U07.1 & COVID-19, virus identified \\
4 & J12.81 & Pneumonia due to SARS-associated coronavirus \\
5 & B97.29 & Other coronavirus as the cause of diseases classified elsewhere \\
\hline
\end{tabular}

ICD code 079.89 (Other specified viral infection) was excluded. This code is mapped to ICD-10 code B34.2 and B97.2, excluded to prevent false positives as it captures all code for many viral infections

ICD, international classification of diseases; LOINC, logical observation identifiers names and codes 